\section{Splicing with super 8}

Pre-mRNA splicing is catalyzed by a dynamic multisubunit complex called the spliceosome that is generated by the ordered interactions of five small nuclear ribonucleoproteins (snRNPs) with a premRNA substrate. Interactions of U2, U5 and U6 snRNPs are critical in forming an active center that coordinates catalysis and substrate repositioning during the two transesterification steps of intron removal. The central region of the U5 snRNP protein Prp8 cross-links with U5 and U6 snRNAs and with all three of the pre-mRNA sites involved in catalysis: the $5^{\prime}$ splice site (5' SS), the $3^{\prime}$ splice site ( $3^{\prime}$ SS) and the branch site. Nagai, Newman and colleagues have now solved the crystal structure of a large fragment of yeast Prp8 in complex with the U5 snRNP-assembly factor Aar2. The structure reveals a large domain comprising two recognizable folds. The larger of these resembles a group II intron reverse transcriptase (RT) and comprises palm, finger and thumb subdomains that form a cleft. The second domain resembles a type II restriction endonuclease (En) and is connected to the RT domain by a linker domain. Importantly, this RT-En domain is juxtaposed with an RNaseH-like domain to form a cavity lined with Prp8 residues that suppress splicing defects caused by pre-mRNA splice-site mutations and which contains branch site- and 5' SS-specific Prp8 cross-linking contacts. The structure has revealed a cavity that can accommodate all the RNA components important for splicing reactions. The structural similarity between Prp8 and group II intron RT has a profound implication: a spliceosomal protein shares a common evolutionary origin with a group II intron-encoded protein. (Nature doi:10.1038/ nature11843, published online 23 January 2013)

$B M$

\section{KRIT-ical interactions}

Cerebral cavernous malformations (CCMs) are vasculature defects that can affect the central nervous system with life-threatening consequences such as strokes or seizures. CCMs are observed in conjunction with an irregular, weak capillary network that lacks proper endothelial layers and subendothelial matrix. A large fraction of CCM-associated mutations arise within the CCM1 gene that encodes KRIT1, a protein whose function is not well understood. KRIT1's ability to bind the integrin cytoplasmic associated protein-1 (ICAP1) - which interacts with the cytoplasmic tail of $\beta 1$-integrin and prevents binding and activation by Talin and Kindlin-suggests a connection between CCMs and defects in cell-adhesion control. Association with KRIT1 prevents ICAP1 binding to $\beta 1$-integrin and therefore indirectly promotes integrin activation. The molecular details of the interactions between KRIT1 and ICAP1 are now illuminated by crystal structures of the KRIT1-ICAP1 and $\beta 1$-integrin-ICAP1 complexes, reported by Boggon and colleagues. Although the N-terminal region of KRIT1 on its own evaded crystallization, coexpression with the predicted PTB domain of ICAP1 allowed structural analysis of the complex, which revealed that the $\mathrm{N}$ terminus of the KRIT1 folds as a Nudix domain, something not predicted from its primary sequence. The interaction surface between KRIT1 and ICAP1 is composed of two distinct sites, one being a canonical NPxY-PTB interaction. The second interaction site was

Written by Angela K. Eggleston, Stéphane Larochelle \& Beth Moorefield unanticipated, but the involved residues on both KRIT1 and ICAP1 are evolutionarily conserved, which points to the likely importance of this interface. Turning to the ICAP1- $\beta 1$-integrin complex, the $\beta 1$ tail is seen to bind ICAP1 in the canonical PTB-binding site, consistent with KRIT1 directly competing with $\beta 1$-integrin for ICAP1 association, a point that is clearly demonstrated by functional analyses. In sum, this study provides a mechanistic understanding of how inactivation of KRIT1, commonly observed in association with CCMs, can result in improper integrin activation, which in turn may contribute to a leaky vasculature. (Mol. Cell doi:10.1016/j.molcel.2012.12.005, published online 10 January 2013)

$S L$

\section{Stressed ribosomes take the slow road}

Molecular chaperones act as sentries within the cell to detect and refold proteins that have become misfolded. When cells are stressed, existing proteins can misfold, constituting a 'proteotoxic' stress that induces upregulation of the chaperones necessary to refold them. Another cellular stress response is the global downregulation of protein translation, previously thought to occur predominantly by inhibition of the initiation stage, which is when most regulation occurs. However, two recent studies, by Qian and colleagues and by Burge and colleagues, show that the ribosome is also controlled at the elongation phase during proteotoxic stress. The authors observed that in stressed cells, most ribosomes undergo extensive but transient pausing at an early stage in elongation, within the first 50-65 codons. This is coincident with a shift in profile from heavy polysomes to lighter polysomes or monosomes. Because the ribosome occludes 30-40 amino acids of the nascent polypeptide chain, the site of pausing suggested that accumulated misfolded proteins could be titrating out ribosome-associated chaperones that lie near the peptide-exit tunnel, such as Hsp70, and that this titration inhibited translation. By various means, this model was confirmed. The mRNAs that show translational pausing encode polypeptides with hydrophobic $\mathrm{N}$ termini, which are preferred ligands for $\mathrm{Hsp} 70$, and the polysomes on those transcripts were associated with 2- to 4-fold less Hsp70. Treatment with an Hsp70 inhibitor led to accumulation of ribosomes at $5^{\prime}$ ends even in the absence of stress. Conversely, moderate overexpression of Hsp70 could alleviate some ribosomal pausing under stress conditions. Heat shock also reduced the association of ribosomal proteins that sit near the exit tunnel (RPL23A) with Hsp70. Supplementation with recombinant chaperones in an in vitro translation system (derived from stressed cells) could reactivate translation. By suppressing translation at both the initiation and elongation levels, the amount of cellular proteins that require refolding by chaperones is reduced. Although these studies indicate that the level of $\mathrm{Hsp} 70$ available for cotranslational association with nascent chains dictates elongation, it is still unclear mechanistically how the absence of chaperones brings translation to a halt. Burge and colleagues suggest that the chaperones regulate the structure of the exit tunnel and note also that lower levels of chaperones at the exit tunnel during cellular stress may result in misfolding of $\mathrm{N}$-terminal hydrophobic patches. (Mol. Cell doi:10.1016/ j.molcel.2012.12.001 and 10.1016/j.molcel.2012.11.028, published online 3 Jan 2013) 